FORMATION Formation emploi

Revue française de sciences sociales

120 | Octobre-Décembre 2012

Parcours de formation : la recomposition des ségrégations

\title{
Quel impact des ségrégations socio-spatiales sur la réussite scolaire au collège?
}

The impact of social segregation on educational achievement

Welchen Einfluss haben sozial-räumliche Segregationen auf den schulischen

Erfolg im Collège (sekundarstuffe)

¿Cuál es el impacto de las segregaciones socioespaciales sobre el éxito en la escuela secundaria?

Danièle Trancart

\section{OpenEdition}

Journals

Édition électronique

URL : http://journals.openedition.org/formationemploi/3761

DOI : $10.4000 /$ formationemploi.3761

ISSN : 2107-0946

Éditeur

La Documentation française

Édition imprimée

Date de publication : 31 octobre 2012

Pagination : $35-55$

ISSN : 0759-6340

Référence électronique

Danièle Trancart, «Quel impact des ségrégations socio-spatiales sur la réussite scolaire au collège ? », Formation emploi [En ligne], 120 | Octobre-Décembre 2012, mis en ligne le 15 février 2013, consulté le 30 octobre 2020. URL : http://journals.openedition.org/formationemploi/3761 ; DOI : https://doi.org/ 10.4000/formationemploi.3761

(c) Tous droits réservés 


\title{
Quel impact des ségrégations socio- spatiales sur la réussite scolaire au collège?
}

\author{
Danièle Trancart \\ Centre d'études de l'emploi
}

Résumé

Quel impact des ségrégations socio-spatiales sur la réussite scolaire au collège?

Les études sur la répartition spatiale des populations font apparaitre une accentuation des ségrégations sociales et scolaires qui se manifeste en particulier par une concentration croissante de la pauvreté dans certains territoires excentrés ou enclavés. Les travaux quantitatifs permettant la mesure de la ségrégation socio-spatiale sont assez rares car le problème soulevé nécessite de construire un ensemble d'indices et de méthodes pertinents. L'objet de cet article est donc de présenter quelques méthodes et indices utilisés dans la mesure de la ségrégation socio-spatiale et de montrer le lien entre ségrégation sociale, concurrence entre établissements et faibles performances scolaires au collège.

Mots clés : Inégalités, inégalité sociale, accès à l'éducation, réussite scolaire, collège, approche locale

Abstract

The impact of social segregation on educational achievement

In recent years the extent of social segregation among French secondary schools is mainly due to a greater social polarization in some urban areas. There have been little studies about social segregation mainly because of the difficulties to build a set of relevant methods and measures.

The aim of this paper is to provide various measures of social and spatial segregation in order to assess the links between segregation, competitiveness and educational achievement.

Key words: Inequalities, social inequality, access to education, success at school, college, local approach

Journal of Economic Literature: I 21

Traduction : Auteurs 
Pour un ensemble d'institutions et de champs de la recherche (la ville, le logement, le travail social...), les dynamiques territoriales font l'objet de travaux depuis plusieurs décennies; cependant, dans le domaine de l'éducation, la place de ces dynamiques est longtemps restée plus modeste. Les difficultés sont nombreuses dès lors qu'il s'agit de mesurer et d'interpréter les différenciations territoriales constatées ; celles-ci peuvent en effet résulter d'une combinaison complexe de facteurs géographiques, historiques, économiques, démographiques et politiques.

Comment la réussite scolaire varie-t-elle selon le lieu de scolarisation, notamment à l'entrée et au sortir du collège ? Comment comprendre les différences constatées ? Quels sont les principaux facteurs sous-jacents aux plus fortes variations ainsi repérées?

Notre texte prend appui sur la recherche "Les inégalités socio-spatiales d'éducation. Processus ségrégatifs, capital social et politiques territoriales », réalisée entre 2002 et 2006 dans le cadre de l'appel à projet interministériel (ministère de l'Education nationale - MEN et Délégation interministérielle à l'aménagement du territoire et à l'attractivité régionale DATAR). Cette recherche a fait l'objet d'une actualisation en 2010 pour la publication de l'ouvrage : Ecole, les pièges de la concurrence. Comprendre le déclin de l'école française (La Découverte "Cahiers libres ", 2010). Elle vise à répondre à ces questions et à celle des ségrégations sociales et scolaires en lien avec la concurrence entre établissements.

La première partie revient sur les enseignements que l'on peut tirer des études empiriques concernant les inégalités scolaires et les ségrégations sociales et scolaires. La ségrégation sociale, définie comme l'inégale distribution des groupes sociaux minoritaires au sein d'unités spatiales (collèges, départements ou académies), peut être vue comme une forme d'inégalité en elle-même ou bien susceptible d'avoir des conséquences négatives sur les résultats scolaires, les attitudes ou les projets des jeunes.

La deuxième partie propose une analyse de la ségrégation sociale dans les collèges publics et les départements français métropolitains. On précisera d'abord quelles caractéristiques sociales il est possible et utile de prendre en compte. Nous examinerons la variété des populations d'élèves puis leur répartition géographique. On verra, en particulier, où sont concentrées les familles d'élèves en situation de précarité, ou au contraire les plus « favorisées » socialement. On s'intéressera ensuite à la façon dont les élèves sont répartis dans des collèges plus ou moins mixtes ou ségrégués socialement selon les départements. Enfin, dans une troisième partie, nous établirons un lien entre l'inégale réussite des départements français et les processus ségrégatifs à l'œuvre. Les derniers résultats du programme PISA $^{1}$ l'ont encore mis en évidence récemment : en France, bien plus que dans la plupart des autres pays, les chances d'acquérir les compétences scolaires sont très liées à l'origine sociale des élèves.

1. Le Programme International de Suivi des Acquis des élèves (PISA) évalue, depuis 2000 et tous les trois ans, ce que les élèves de différents pays ont appris à 15 ans dans différents domaines (mathématiques, compréhension, écrit et sciences). 


\section{La ségrégation sociale est une source d'inégalités scolaires}

La sociologie de l'éducation s'est structurée, durant les 60/70, autour de la relation école/ société par l'étude des liens entre inégalités sociales et inégalités scolaires. Même si certaines recherches introduisent la notion d'inégalités géographiques du système d'enseignement, mettant en question son caractère unifié, cette période est davantage marquée par une lecture centralisatrice de l'école, privilégiant la notion d'appartenance de classe. Il faut attendre les années 80 pour que se développent des travaux qui mobilisent la dimension spatiale, en lien avec un ensemble de transformations politiques et institutionnelles qui justifient une interrogation plus systématique des relations entre éducation et enjeux locaux (décentralisation, territorialisation, assouplissement de la carte scolaire...) mais ces travaux sont de nature essentiellement qualitative (par exemple : Barthon, 1997 ; Broccolichi, Van Zanten, 1997 ; Van Zanten, 2000).

Dans les années 90, un certain nombre de sociologues, de géographes et d'économistes proposent des analyses empiriques systématiques de la ségrégation socio-spatiale.

La typologie réalisée à chaque recensement par l'Insee (Tabard, 1993 ; 2002), à partir de l'organisation socio-économique des quartiers et des communes ${ }^{2}$ de la métropole parisienne, permet d'analyser les inégalités territoriales. Celles-ci se sont creusées depuis le recensement de 1990, et à partir de 1999 "le chômage structure le territoire".

En comparant les recensements de 1975 et 1990 pour étudier le lien entre ségrégation scolaire et résidentielle (Rhein, 1997), l'auteur conclut que " la force et l'augmentation de la relation entre catégorie socioprofessionnelle, nationalité et nombre d'enfants confirment l'apparition de puissants processus ségrégatifs".

Les travaux sur la division sociale de l'espace francilien (Préteceille, 2003 ; 2006) montrent que l'analyse des ségrégations et de leur évolution diverge en fonction des choix sur les variables descriptives de l'analyse, des découpages spatiaux et des méthodes statistiques proprement dites. Néanmoins, l'auteur observe un mouvement général du profil des IRIS (Ilots Regroupés d'Information Statistique) ${ }^{3}$, entre 1990 et 1999, vers une progression des statuts professionnels des populations. En dépit de cette tendance générale, les écarts entre les types d'espaces extrêmes se sont accentués ; il y a donc eu une bipolarisation des profils socioprofessionnels des espaces franciliens. En particulier, l'auteur note un recul marqué des catégories supérieures et moyennes dans des espaces populaires et un accroissement de ces mêmes catégories dans les espaces favorisés.

2. La typologie est construite à partir de la distribution des hommes personnes de référence dans les unités géographiques de résidence selon leur position d'emploi (croisement de la PCS - profession et catégorie sociale -, la situation par rapport à l'emploi et la branche d'activité).

3. Dans le cadre du recensement de la population, l'INSEE utilise un découpage du territoire de taille homogène, appelé IRIS. 
Dans une tribune du 23 octobre 2004 à propos du « ghetto français », Eric Maurin (2004) souligne que : "Le débat démocratique a certainement avancé ces dernières années puisque le terme de "ghetto" ou de "ségrégation" n’y sont plus tabous: face à la réalité des quartiers, la pudeur et le long cortège des euphémismes d'hier ne sont plus de mise. Mais un nouveau pas doit être franchi: alors que beaucoup considèrent encore que les déchirements de la ville affectent essentiellement une minorité d'exclus, il va falloir accepter l'idée que les mécanismes de la ségrégation traversent toute la société et non seulement ses franges. "

La ségrégation sociale est donc un fait mais est-elle source d'inégalités scolaires ?

La ségrégation sociale mise en évidence à l'échelle des quartiers se diffuse dans le milieu scolaire. L'espace scolaire parisien apparaît même plus hiérarchisé et plus fragmenté du fait des pratiques d'évitement scolaire (Poupeau, François, 2008).

Dans une étude sur l'évolution de la ségrégation sociale dans les collèges parisiens publics et privés de 2005 à 2008 (Merle, 2010), l'auteur constate une augmentation de la ségrégation avec l'assouplissement de la carte scolaire.

En France, les travaux sur la ségrégation sociale ou les processus ségrégatifs en lien avec les inégalités scolaires sont plus rares. Ils rendent compte des liens entre la hiérarchisation croissante entre établissements, entre classes au niveau de la composition sociale, ethnique ou des offres d'enseignement (langues, options...), d'une part, et les inégalités d'acquisitions entre élèves, d'autre part (Broccolichi, 2011). Ces recherches autour du "school mix » qui évaluent, plus généralement, l'impact de la composition du public scolaire sur les acquis, ont fait l'objet de quelques travaux ${ }^{5}$.

Dans une recherche sur les progressions d'élèves d'établissement socialement contrastés (Duru-Bellat, Landrier-Le Bastard, Piquée, 2004), les auteurs montrent que l'effet du " school mix » apparait systématique mais quantitativement modeste.

Citons une autre recherche centrée sur l'impact de la ségrégation ethnique (" ethnic mix ») dans la communauté urbaine de Bordeaux (Felouzis, 2005). Cet impact négatif, mais globalement relativement faible, est surtout significatif dans les collèges où le pourcentage d'élèves étrangers est supérieur à $20 \%$. L’offre d'enseignement et surtout le niveau de qualification des enseignants et leur expérience (en distinguant les enseignants débutants et ceux ayant au moins 2 ans d'expérience) exercent également une influence sur les acquisitions des élèves (Duru-Bellat et al., 2004).

L'article proposé s'inscrit dans le prolongement de travaux réalisés sur les disparités à l'échelle nationale entre collèges publics français (Trancart, 1998) et non sur une agglo-

4. Il s'agit de l'évitement de l'établissement du secteur attribué par la carte scolaire. Cet évitement a surtout pour effet d'accentuer les polarisations existantes, c'est-à-dire de renforcer les écarts entre le Paris favorisé et le Paris défavorisé.

5. Pour une synthèse de ces travaux, voir Duru-Bellat, 2004. 
mération particulière. Les questions relatives aux échelles d'analyse et à la mesure de la ségrégation sont également discutées. Enfin, nous proposons une analyse du lien entre inégalités scolaires et ségrégations sociales, au collège et à l'échelle départementale.

\section{Une grande hétérogénéité sociale des territoires}

La question de l'égalité des chances représente un enjeu majeur des politiques éducatives aujourd'hui. Dans le cadre de cette réflexion, la ségrégation sociale, définie comme l'inégale distribution des groupes sociaux minoritaires au sein d'unités spatiales (collèges, départements ou académies), peut être vue comme une forme d'inégalité en elle-même ou bien susceptible d'avoir des conséquences négatives sur les résultats scolaires, les attitudes ou les projets des jeunes.

Nous proposons, dans cette partie, plusieurs mesures de la ségrégation sociale mais il convient, tout d'abord, de présenter les données sociales utilisées (encadré 1) ainsi que leur distribution. L’année scolaire 2001-2002 sert de référence à ces analyses car il s’agit de l'année de démarrage de la recherche. Les évolutions concernent des années antérieures et postérieures en couvrant une période d'une quinzaine d'années.

\subsection{Des disparités sociales entre les collèges publics français}

Nous nous sommes intéressé, dans un premier temps, au niveau collège et surtout $6^{\text {ème }}$. Ce dernier présente en effet l'avantage de prendre en compte la quasi-totalité d'une génération, sans les biais liés aux orientations qui s'amorcent un peu avant la fin du collège et se diversifient surtout après (en lycée général ou technologique, en lycée professionnel, lycée agricole ou par la voie de l'apprentissage).

En fonction des connaissances établies sur les inégalités de réussite scolaire, nous avons d'abord distingué les catégories sociales " très favorisées ", " très défavorisées " et " ouvriers ». La proportion d'élèves étrangers et la proportion d'élèves boursiers de sixième sont, ensuite, venues compléter l'analyse. L'encadré $\mathbf{1}$ fournit quelques précisions supplémentaires sur les sources, la sélection et la définition de ces catégories.

On constate que la composition sociale des collèges est très diverse (Tableau 1). Certains collèges présentent de fortes concentrations de populations issues de milieu "populaire ", tandis que d'autres concentrent majoritairement des populations aisées. Par exemple, si $10 \%$ de collèges accueillent moins de $16 \%$ d'élèves issus de la catégorie "ouvrier ", à l'autre extrême, $10 \%$ de collèges en accueillent plus de $55 \%$. On remarque que la composition sociale des collèges en ZEP (zone d'éducation prioritaire) recouvre bien celle des $10 \%$ de collèges les plus défavorisés socialement. 
Encadré 1

\section{Données et variables prises en compte}

Les données proviennent de la DEPP (Direction de l'évaluation de la prospective et de la performance), ministère de l'Education nationale, dans le cadre de l'appel d'offre MEN-DATAR (ministère de l'Education nationale- Délégation interministérielle à l'aménagement du territoire et à l'attractivité régionale) en 2002, cité précédemment. Elles sont complétées par des bases associées à des travaux plus anciens réalisés au cours des années 90 mais aussi au cours d'années plus récentes, suite à une actualisation de nos travaux. Finalement, les données couvrent une période d'une quinzaine d'années, de 1993 à 2009. Elles fournissent des informations sur l'origine sociale des élèves de collèges publics de France métropolitaine, les caractéristiques des établissements ainsi que les moyennes des épreuves aux évaluations de sixième et de troisième.

\section{L'origine sociale}

Si l'on veut tenir compte assez finement de la répartition des fractions de population particulièrement fragiles sur le plan des conditions de vie et des chances scolaires, il est essentiel de ne pas s'en tenir aux regroupements habituels des données, surtout ceux concernant la macro catégorie appelée « défavorisée » (catégorie d'appartenance de la personne responsable de l'élève) qui regroupe les ouvriers, retraités employés ou ouvriers, chômeurs n'ayant jamais travaillé et personnes sans activité professionnelle.

Par ailleurs, les résultats détaillés aux évaluations en 6ème et au diplôme national du brevet (DNB) nous ont incités à distinguer tout particulièrement les groupes sociaux dont les résultats sont les plus décalés par rapport à la moyenne nationale. D'une part, les catégories « très favorisées » (professions intellectuelles supérieures, cadres, chefs d'entreprise de plus de 10 salariés et enseignants), et d'autre part les « très défavorisées ». Cette dernière catégorie comprend les chômeurs n'ayant jamais travaillé et les personnes sans activité professionnelle, ainsi que les familles pour lesquelles les établissements n'ont d'autres indications que " chômage », « ne travaille pas » ou « invalide ». Elle correspond à près de $9 \%$ de la population scolaire de 6ème, chaque année, et constitue le groupe dont les espérances scolaires sont incontestablement les plus faibles. On ne peut donc négliger le fait que sa proportion puisse varier considérablement selon les territoires. Finalement, nous avons retenu trois groupes sociaux contrastés : les " très défavorisés », les « ouvriers » (ouvriers, retraités employés ou ouvriers) et les « très favorisés», la catégorie « défavorisée » classique regroupant les « ouvriers » et les « très défavorisés ».

\section{La part d'élèves boursiers}

La prise en compte de la part d'élèves boursiers en moyenne dans les collèges vient compléter les données sociales précédentes en introduisant un indicateur économique.

\section{La part d'élèves étrangers}

La proportion d'élèves de sixième de nationalité étrangère rend compte également de l'environnement social du collège. Les données nationales montrent bien les interrelations entre certaines variables : les élèves étrangers se situent dans des environnements différents de celui des élèves français, et leurs scolarités sont affectées de retards et d'échecs plus importants

Les caractéristiques sociales des collèges ont été étudiées en 2002, année de démarrage de la recherche, mais nous avons vérifié que les résultats étaient peu modifiés pour les années postérieures. Les données relatives aux épreuves d'évaluation sixième et troisième étaient connues, au moment du démarrage de la recherche, pour les années 2001-2002 puis 2003-2004. Nous avons alors calculé la moyenne de ces épreuves pour ces deux années, afin de lisser les résultats. 
Tableau 1

Disparités sociales entre collèges publics de France métropolitaine en \%

\begin{tabular}{|l|c|c|c|c|}
\hline & $\begin{array}{c}\text { Pourcentage } \\
\text { d'élèves de } \\
\text { catégorie « très } \\
\text { défavorisée » }\end{array}$ & $\begin{array}{c}\text { Pourcentage } \\
\text { d'élèves de } \\
\text { catégorie } \\
\text { « ouvrier » }\end{array}$ & $\begin{array}{c}\text { Pourcentage } \\
\text { d'élèves boursiers }\end{array}$ & $\begin{array}{c}\text { Pourcentage } \\
\text { d'élèves étrangers }\end{array}$ \\
\hline $\begin{array}{l}\text { Moyenne } \\
\text { (près de 4900 collèges) }\end{array}$ & 9 & 37 & 25 & 5 \\
\hline $\begin{array}{l}\text { Premier décile (10\% } \\
\text { des collèges) }\end{array}$ & 2 & 16 & 9 & 0 \\
\hline $\begin{array}{l}\text { Dernier décile (10\% } \\
\text { des collèges) }\end{array}$ & 18 & 55 & 44 & 13 \\
\hline $\begin{array}{l}\text { Collèges en ZEP }(*) \\
(16,6 \% \text { des collèges) }\end{array}$ & 17 & 47 & 45 & 12 \\
\hline
\end{tabular}

Source et champ : DEPP, ministère de l'Education nationale, collèges publics année 2001-2002. Calculs de l'auteure.

Note de lecture : En moyenne, on compte $37 \%$ d'élèves issus de la catégorie « ouvrier », $10 \%$ de collèges publics accueillent moins de $16 \%$ (premier décile) d'élèves de cette catégorie alors quà l'autre extrême, $10 \%$ de collèges en accueillent plus de $55 \%$ (dernier décile).

$\left.{ }^{*}\right)$ ZEP : zone d'éducation prioritaire.

\subsection{L'analyse de la ségrégation sociale à l'aune de typologies ou d'indices de ségrégation}

La distribution inégale des groupes sociaux sur le territoire nous a conduits à nous interroger sur le concept de ségrégation spatiale. Elle est le résultat de plusieurs processus (marché immobilier, localisation des entreprises, volonté des habitants de se rapprocher de certains groupes sociaux etc.). Nous pensons que la concentration de difficultés dans un territoire doit avoir des conséquences négatives sur les résultats scolaires. Cette ségrégation sociale risque également de produire des effets directs sur les attitudes, les projets et les ambitions scolaires des jeunes et sur les choix de carrière des enseignants.

Rares sont les travaux quantitatifs permettant de mesurer l'état de la mixité sociale des collèges à l'échelle nationale et d'en apprécier l'évolution. En effet, la disponibilité de sources homogènes et le problème quantitatif soulevé nécessitent de construire un ensemble d'indices pertinents. Globalement, il existe deux types d'approches : les unes s'appuient sur la construction de typologies à partir de plusieurs indicateurs (approche multidimensionnelle) ; les autres sont fondées sur le calcul d'indices de ségrégation (approche unidimensionnelle). On reconnaît une spécificité méthodologique française dans le premier cas et anglo-saxonne dans le deuxième.

\subsubsection{Une typologie qui révèle la diversité sociale des collèges publics français}

À partir des données socio-économiques décrites précédemment (part de boursiers dans le collège, part d'élèves issus de catégories sociales "très favorisées ", part d'élèves dont le responsable appartient à la catégorie " très défavorisée ", part d'élèves dont le responsable 
appartient à la catégorie « ouvriers ", part d'élèves boursiers, et part d'élèves étrangers), une typologie (obtenue par une Classification Ascendante Hiérarchique) des collèges en six groupes a été construite.

Cette typologie met en évidence deux groupes de collèges très défavorisés (Figure 1, Groupe E et Groupe F, 12 \% des collèges au total). En moyenne, ils accueillent près de $45 \%$ d'enfants d'ouvriers (37\% en moyenne), une très forte surreprésentation d'élèves boursiers (60\% dans le groupe $\mathrm{F}$ et $45 \%$ dans le groupe $\mathrm{E}$, contre $25 \%$ en moyenne) et d'élèves étrangers (près de $10 \%$ dans le groupe $\mathrm{F}$ et $22 \%$ dans le groupe $\mathrm{E}$, contre $5 \%$ en moyenne). À l'opposé, $12 \%$ de collèges (Groupe A) présentent une forte surreprésentation de catégories très favorisées (plus de $40 \%$ contre $15 \%$ en moyenne). Les trois autres groupes $\mathrm{B}, \mathrm{C}$ et $\mathrm{D}$ sont respectivement assez favorisés (32\% de l'ensemble des collèges), ouvriers (27\% de l'ensemble), et plutôt défavorisés (27\% de l'ensemble) (Figure 1).

Figure 1

Profil des classes de la typologie des collèges publics de France métropolitaine (\%)

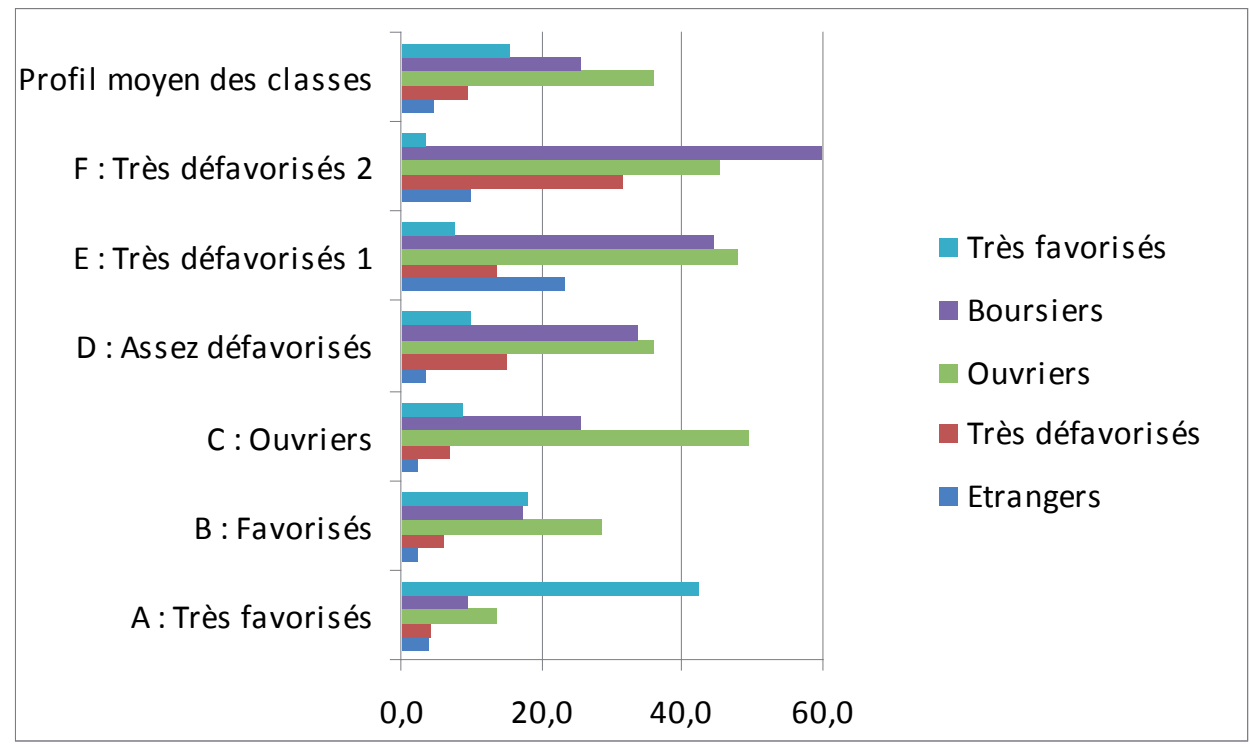

Source et champ : DEPP, ministère de l'Education nationale, collèges publics année 2001-2002. Calculs de l'auteure.

Note de lecture : Dans la classe A des collèges dits « très favorisés », la distribution sociale des élèves est la suivante : plus de $40 \%$ d'élèves issus des catégories très favorisées, $8 \%$ d'élèves boursiers, $12 \%$ d'enfants d'ouvriers, $3 \%$ d'élèves issus de catégories très défavorisées (la personne responsable de l'élève est chômeur ou inactif) et $3 \%$ d'élèves étrangers. 
Notre travail nous a conduits à préférer l'échelle départementale à l'échelle académique car d'importantes différences de situations et de résultats entre départements d'une même académie apparaissent.

Les départements ne se distribuent pas au hasard dans les groupes décrits précédemment. L'importance relative de chacune des six catégories de collèges au sein de chaque département permet de faire apparaitre des disparités interdépartementales aussi bien qu'intradépartementales. Par exemple, Paris se caractérise bien par une concentration des collèges sur les catégories extrêmes ( $46 \%$ très favorisés (type $\mathrm{A}$ ) et $30 \%$ très défavorisés (type $\mathrm{E}$ ) avec une forte surreprésentation d'élèves étrangers). Ce profil contrasté de départements est la traduction des inégalités sociales observées sur ces territoires. La ségrégation sociale y est plus forte qu'ailleurs.

Cette analyse rend bien compte de la donne sociale d'un territoire ainsi que de son degré d'hétérogénéité ; cependant, elle se prête mal à une analyse diachronique à partir d'indicateurs synthétiques que nous présentons ci-dessous.

\subsubsection{Indices de ségrégation}

Nous présenterons, dans un premier temps, les indices d'inégalité le plus souvent utilisés dans l'analyse de la ségrégation socio-spatiale à partir de plusieurs variables descriptives, et ensuite, nous proposerons un indice global unique pour analyser la tonalité sociale d'un territoire.

\section{- Indices d'inégalité pour mesurer la sur ou sous-représentation spatiale d'un groupe}

Le calcul d'un indice de ségrégation constitue une autre approche de l'analyse des disparités et présente l'avantage d'une analyse diachronique.

Parler de ségrégation suppose que deux groupes d'élèves ne sont pas scolarisés dans les mêmes établissements et sont donc séparés les uns des autres. Ces différents groupes d'élèves peuvent être définis à partir des variables sociales disponibles et décrites précédemment : élèves de $6^{\text {ème }}$ appartenant à des catégories sociales très défavorisées ou non, élèves étrangers ou non, ou plus généralement appartenant à un groupe minoritaire ou non.

De nombreux indices sont utilisés dans les travaux portant sur les problèmes de ségrégation scolaire ou sociale mais aucun d'eux ne fait vraiment l'unanimitét. Les indices d'inégalité, les plus utilisés, mesurent la sur ou sous-représentation d'un groupe dans les différentes unités spatiales. Un groupe est ségrégué s'il est inégalement réparti dans les unités spatiales ; l'indice calculé étant alors élevé. Il peut s’agir d'un indice inter-groupes (" écart " entre deux groupes) ou uni-groupe (" écart " entre un groupe et la situation moyenne idéale).

6. Une analyse des résultats de ces travaux (essentiellement anglo-saxons) et des polémiques suscitées a été conduite par D. Meuret et alii (2001). 
L'indice de Gini constitue une alternative aux précédents indices et est couramment employé dans les travaux sur les inégalités de revenu ou patrimoine.

Pour chacune des variables permettant de décrire la composition sociale des collèges, et pour chaque année étudiée, nous avons calculé et comparé les indices de ségrégation, entre 1993 et 2009 (Tableaux 2 à 4) :

- L'indice de dissimilarité « DS » (Duncan, 1955), qui représente la proportion d'élèves du groupe minoritaire qu'il faudrait déplacer pour obtenir un pourcentage d'élèves du groupe minoritaire identique dans chaque collège. C'est un indice uni- groupe qui compare la répartition d'un groupe minoritaire à la répartition moyenne et est utilisé dans l'enquête PISA. Cet indice est compris entre 0 et 1 . Il vaut 0 en situation idéale et 1 dans la situation la plus inégalitaire.

- L'indice de ségrégation « $S$ » est une variante du précédent. C'est un indice intergroupe, compris entre 0 et 1 , qui compare la répartition du groupe minoritaire au groupe majoritaire.

- L'indice synthétique de Gini (G), également compris entre 0 et 1 . Cet indice mesure le degré d'inégalité d'une distribution. L'égalité parfaite (répartition égale du groupe minoritaire) correspond à un indice égal à zéro alors que l'inégalité totale correspond à une valeur égale à 1 .

Le coefficient de variation CV (indicateur de dispersion qui rapporte l'écart-type à la moyenne et exprimé ici en pourcentage) complète l'analyse. Plus la valeur de cet indice est élevée, plus la dispersion par rapport à la moyenne est grande.

Tableau 2

Evolution des indices de ségrégation sociale calculés à partir de la proportion d'élèves appartenant à des catégories socialement défavorisées ${ }^{*}$ )

\begin{tabular}{|l|c|c|c|c|c|}
\hline Année & \% élèves défavorisés & $\begin{array}{c}\text { Indice de } \\
\text { dissimilarité } \\
\text { (DS) }\end{array}$ & $\begin{array}{c}\text { Indice de } \\
\text { ségrégation (S) }\end{array}$ & $\begin{array}{c}\text { Indice de Gini } \\
\text { (G) }\end{array}$ & $\begin{array}{c}\text { Coefficient } \\
\text { de variation } \\
\text { (CV) }\end{array}$ \\
\hline $1993-1994$ & $(\mathbf{e n} \%)$ \\
\hline $1999-2000$ & $44 \%$ & 0,28 & 0,16 & 0,22 & 39 \\
\hline $2001-2002$ & $44 \%$ & 0,28 & 0,16 & 0,22 & 38 \\
\hline $2003-2004$ & $43 \%$ & 0,28 & 0,16 & 0,22 & 38 \\
\hline $2008-2009$ & $44 \%$ & 0,29 & 0,16 & 0,23 & 40 \\
\hline
\end{tabular}

Source et champ : DEPP, scolarité, collèges publics, France métropolitaine, calculs de l'auteure.

$(*)$ : La proportion d'élèves défavorisés s'obtient en ajoutant la proportion d'enfants d'ouvriers à la proportion d'enfants issus de catégories très défavorisées (voir encadré 1 ).

(1) Le coefficient de variation rapporte l'écart-type à la moyenne. II peut s'exprimer en \%. 
Tableau 3

Evolution des indices de ségrégation sociale calculés à partir de la proportion d'élèves étrangers

\begin{tabular}{|l|c|c|c|c|c|}
\hline \multicolumn{1}{|c|}{ Année } & $\begin{array}{c}\text { \% élèves } \\
\text { étrangers (1) }\end{array}$ & $\begin{array}{c}\text { Indice de } \\
\text { dissimilarité } \\
\text { (DS) }\end{array}$ & $\begin{array}{c}\text { Indice de } \\
\text { ségrégation (S) }\end{array}$ & $\begin{array}{c}\text { Indice de Gini } \\
\text { (G) }\end{array}$ & $\begin{array}{c}\text { Coefficient de } \\
\text { variation (CV) } \\
\text { (en \%) }\end{array}$ \\
\hline $1993-1994$ & $8 \%$ & 0,48 & 0,43 & 0,57 & 127 \\
\hline $1999-2000$ & $6 \%$ & 0,50 & 0,48 & 0,61 & 140 \\
\hline $2001-2002$ & $5,6 \%$ & 0,50 & 0,475 & 0,62 & 143 \\
\hline $2003-2004$ & $4 \%$ & 0,53 & 0,51 & 0,66 & 152 \\
\hline $2008-2009$ & $3 \%$ & 0,61 & 0,59 & 0,76 & 198 \\
\hline
\end{tabular}

Source et champ : DEPP, scolarité, collèges publics, France métropolitaine, calculs de l'auteure.

(1) On calcule, pour chaque collège et pour chacune des 5 variables, un écart centré réduit en retranchant à la valeur initiale d'un collège, la moyenne et en divisant par l'écart-type, puis on effectue la somme algébrique des écarts obtenus en prenant soin de comptabiliser ces écarts sur une même échelle. Ce type d'indice a déjà été utilisé dans des travaux précédents (Trancart, 1998).

Tableau 4

Evolution des indices de ségrégation sociale calculés à partir de la proportion d'élèves « très défavorisés »

\begin{tabular}{|l|c|c|c|c|c|}
\hline Année & $\begin{array}{c}\text { \% élèves très } \\
\text { défavorisés }\end{array}$ & $\begin{array}{c}\text { Indice de } \\
\text { dissimilarité } \\
\text { (DS) }\end{array}$ & $\begin{array}{c}\text { Indice de } \\
\text { ségrégation (S) }\end{array}$ & $\begin{array}{c}\text { Indice de Gini } \\
\text { (G) }\end{array}$ & $\begin{array}{c}\text { Coefficient de } \\
\text { variation (CV) } \\
\text { (en \%) }\end{array}$ \\
\hline $1993-1994$ & $10,4 \%$ & 0,27 & 0,24 & 0,34 & 66 \\
\hline $1999-2000$ & $9,5 \%$ & 0,30 & 0,27 & 0,38 & 76 \\
\hline $2001-2002$ & $9,6 \%$ & 0,31 & 0,28 & 0,38 & 78 \\
\hline $2003-2004$ & $9,8 \%$ & 0,33 & 0,30 & 0,42 & 86 \\
\hline $2008-2009$ & $8,1 \%$ & 0,34 & 0,31 & 0,44 & 95 \\
\hline
\end{tabular}

Source et champ : DEPP, scolarité, collèges publics, France métropolitaine, calculs de l'auteure.

Lanalyse permet, quel que soit l'indice retenu, de conclure à une légère accentuation des disparités entre collèges pour la proportion d'élèves étrangers et " très défavorisés " et à une stabilité de la part des élèves appartenant à des catégories défavorisées. De plus, on peut noter que les disparités concernant la part d'élèves étrangers sont plus fortes que les autres. La polarisation entre établissements est donc plus importante si l'on retient cette variable. Ces résultats sont conformes à ceux d'autres recherches (Préteceille, 2009). Lauteur montre que la ségrégation ethnique, dans la métropole parisienne, augmente et est plus marquée que la ségrégation sociale. Cette concentration plutôt accrue peut 
s'expliquer par plusieurs phénomènes : les mesures d'assouplissement de la carte scolaire, la mise en concurrence des établissements et, surtout, les évolutions concernant les espaces urbains avec des zones ghettos.

\section{- Indice global de précarité, pour mesurer la tonalité sociale d'un territoire}

L'analyse précédente ne conduit pas à un indicateur unique de ségrégation sociale. Pour une approche synthétique, et à partir des variables sociales étudiées, nous avons calculé un indice global de précarité, indice d'autant plus positif que la donne socio-économique ${ }^{7}$ d'un territoire est défavorable et d'autant plus négatif à l'inverse. L'indice est nul pour un territoire dans une situation moyenne sur chaque indicateur (proportion d'ouvriers, proportion d'élèves très défavorisés, d'élèves boursiers, d'élèves étrangers et d'élèves très favorisés).

Les départements dont la tonalité sociale est très défavorisée (indice supérieur à 3) se situent essentiellement dans le nord de la France (Figure 2) : Nord, Pas De Calais, Ardennes et Somme, sans oublier la Seine-Saint-Denis qui s'avère le plus défavorisé sur la plupart des critères sociaux (et le seul département d'Ile-de-France en situation défavorisée) ; la Corse du sud fait figure d'exception au sud, mais on peut noter une tonalité sociale défavorisée (indice compris entre 2 et 3) dans cinq autres départements du sud méditerranéen : on y trouve autant de "très défavorisés " que dans le Nord de la France (résultats conformes à la géographie du chômage) mais moins d' " ouvriers " et un peu plus de " très favorisés ».

À l'autre extrême (indice négatif, inférieur à -2), les trois départements qui comptent les plus fortes proportions de cadres et de chefs d'entreprise sont situés en Ile-de-France (Yvelines, Hauts-de-Seine et Essonne), et nous verrons que les résultats scolaires y sont plutôt décevants, comme dans toute cette région. En dehors de l'Ile-de-France, seule région à dominante vraiment favorisée, c'est principalement dans le Sud-ouest, dans les Alpes et en Bretagne qu'on trouve d'assez fortes proportions de familles socialement favorisées (indice compris entre -1 et -2 ).

7. On calcule pour chaque collège et pour chacune des 5 variables, un écart centré réduit en retranchant à la valeur initiale d'un collège, la moyenne et en divisant par l'écart-type, puis on effectue la somme algébrique des écarts obtenus en prenant soin de comptabiliser ces écarts sur une même échelle. Ce type d'indice a déjà été utilisé dans des travaux précédents (Trancart, 1998). 


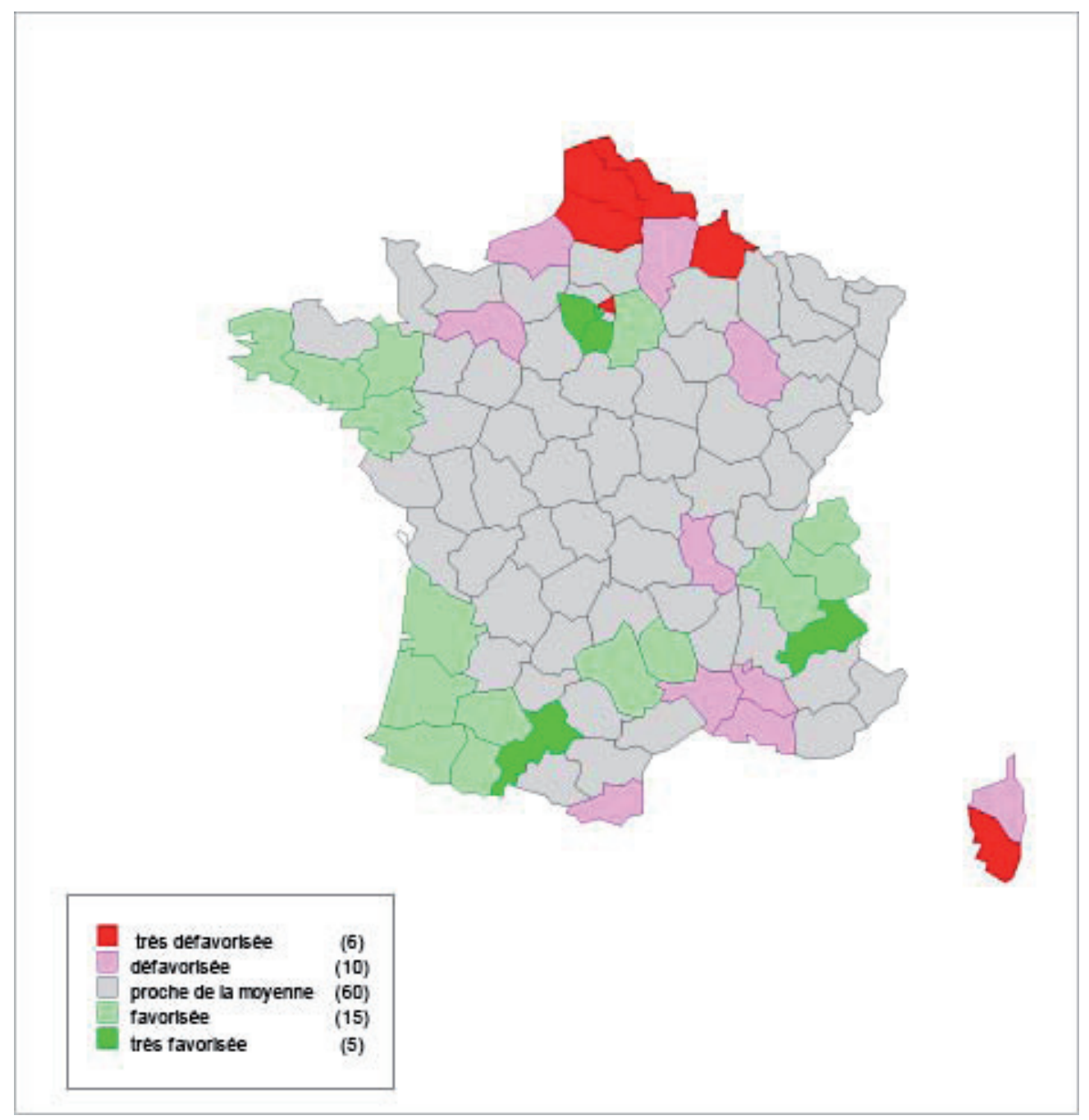

Source et champ : DEPP, scolarité, collèges publics, années 2001-2002 et 2003-2004, France métropolitaine, calculs de l'auteure Note de lecture : 6 départements sont très défavorisés : La Seine-Saint-Denis, le Nord, le Pas-de-Calais, la Somme, les Ardennes et la Corse du Sud.

Quand des départements présentent la même tonalité sociale globale, ils peuvent néanmoins présenter de grandes différences dans la répartition des élèves. Dans les territoires les moins urbanisés, on constate souvent peu de différences entre collèges au niveau de l'origine sociale des élèves, tandis que les collèges sont beaucoup plus profilés et hiérarchisés socialement dans les territoires plus urbanisés et plus ségrégués. Ces ségrégations 
(mesurées ici par l'écart-type de l'indice global de précarité, voir tableau 5) perceptibles au niveau des collèges reflètent en partie les ségrégations résidentielles, tout en étant souvent amplifiées par les pratiques de scolarisation hors secteur. Il n'est donc pas surprenant qu'elles soient les plus marquées en Ile-de-France (écart-type proche de 5) mais aussi dans le Sud Est méditerranéen (écart-type supérieur à 4), autre zone géographique très urbanisée où l'on trouve des collèges " très favorisés " et des collèges " très défavorisés " dans des villes ou des quartiers séparés (tableau 5). À l'opposé, les départements les moins ségrégués, tels que les Landes, les Hautes-Alpes, les Alpes de Haute-Provence, l'Aveyron, la Haute Loire, le Lot, le Tarn et Garonne sont très ruraux (écart-type proche de 1).

Tableau 5

Contrastes entre les départements les plus ou les moins ségrégués

\begin{tabular}{|c|c|c|c|}
\hline \multirow{2}{*}{ Département } & \multirow{2}{*}{ Académie } & \multicolumn{2}{|c|}{ Indice de précarité } \\
\hline & & Moyenne & Ecart-type \\
\hline \multicolumn{4}{|l|}{ Départements les plus ségrégués } \\
\hline 078 Yvelines & Versailles & $-3,4$ & 5,1 \\
\hline 013 Bouches du Rhône & Aix-Marseille & 1,3 & 5,1 \\
\hline 069 Rhône & Lyon & 0,2 & 4,9 \\
\hline 075 Paris & Paris & $-0,8$ & 4,8 \\
\hline 084 Vaucluse & Aix-Marseille & 2,1 & 4,6 \\
\hline 092 Hauts-de-Seine & Versailles & $-2,3$ & 4,5 \\
\hline 034 Héraut & Montpellier & 0,4 & 4,4 \\
\hline \multicolumn{4}{|c|}{ Départements les moins ségrégués } \\
\hline 040 Landes & Bordeaux & $-1,3$ & 1,4 \\
\hline 005 Hautes-Alpes & Aix-Marseille & $-2,1$ & 1,4 \\
\hline 004 Alpes de Haute Provence & Aix-Marseille & $-1,0$ & 1,3 \\
\hline 012 Aveyron & Toulouse & $-1,2$ & 1,1 \\
\hline 043 Haute Loire & Clermont & $-0,3$ & 1,0 \\
\hline 046 Lot & Toulouse & $-0,9$ & 0,9 \\
\hline 082 Tarn et Garonne & Toulouse & 0,4 & 0,7 \\
\hline
\end{tabular}

Source et champ : DEPP, scolarité, collèges publics, moyenne des années 2001-2002 et 2003-2004, France métropolitaine, calculs de l'auteure. Note de lecture : Le département des Yvelines a un indice de précarité dont la moyenne est fortement négative (donne sociale favorisée) et l'écart-type (mesure de dispersion) très élevé et égal à 5,1 (forte polarisation ou ségrégation entre collèges du département).

Au-delà d'un certain degré, les ségrégations entre collèges, à l'échelle départementale, témoignent du déficit de l'école à promouvoir la mixité sociale et s'avèrent préoccupante pour l'équilibre du système éducatif. Cette ségrégation est souvent associée à des inégalités 
au niveau de la qualité des services publics, dont l'école. Cette dimension est d'ailleurs très présente dans les débats de la politique de la ville et dans l'analyse des phénomènes d'échec scolaire et de violence chez les jeunes.

Peu étudiées en France, les relations entre ségrégation sociale et inégalités de résultats ont néanmoins fait l'objet de recherches comparatives en éducation ${ }^{8}$. Un des résultats les mieux établis est que les pays où les établissements sont les plus ségrégués socialement sont aussi les pays où les inégalités d'acquis scolaires sont les plus marquées entre élèves. Nos propres comparaisons entre départements français conduisent à des résultats similaires, et nous nous attacherons à préciser l'ampleur de ces inégalités, mais seulement après avoir comparé plus globalement les moyennes de performances départementales.

\section{Des performances scolaires inégales selon les conditions de scolarisation}

Dans un premier temps, le but est de repérer les départements où la moyenne des performances des élèves aux épreuves standardisées de sixième est nettement supérieure, ou au contraire inférieure, à la moyenne prédite en fonction de la composition sociale des collégiens du département. Précisons que l'échelle des collèges pour l'analyse des performances des élèves est biaisée car elle ne prend pas en compte "leur position sur un marché local dans des territoires plus ou moins urbanisés", c'est-à-dire leur inégale sélectivité (Broccolichi, 2011). L'échelle départementale n'est pas sensible à ces biais et permet de mieux mettre en évidence les différences liées aux conditions de scolarisation.

À l'aide d'un modèle de régression simple, nous avons calculé le résultat attendu à l'évaluation de Gème (c'est-à-dire la valeur prédite par le modèle en fonction du seul indice global de précarité) $)^{9}$. L'écart entre le résultat observé et le résultat attendu représente la sur-réussite (si l'écart est positif) ou sous-réussite dans le cas contraire ${ }^{10}$.

Dans plus de la moitié des départements, les résultats constatés diffèrent peu des résultats prédits en fonction de l'indicateur utilisé pour mesurer la tonalité sociale du département (moins de $2 \%$ d'écart). Autrement dit, dans ces départements, les acquis scolaires des élèves sont liés à leur origine sociale d'une façon proche du lien observé à l'échelle nationale.

En revanche, les écarts entre résultats constatés et prévus sont très significatifs et surprenants au premier abord dans une vingtaine d'autres départements. Les huit départements où ces écarts sont les plus élevés sont en "sous-réussite " : les résultats constatés y sont nettement inférieurs aux résultats prévus d'après les caractéristiques sociales des familles

8. Pour une synthèse de ces travaux, voir Duru-Bellat, 2004.

9. Des résultats comparables sont obtenus avec les épreuves du DNB (diplôme national du brevet).

10. Rappelons que nous ne disposons pas de données individuelles relatives aux évaluations en sixième. 
d'élèves. Sur la carte (Figure 3), on voit qu'ils se situent tous dans le bassin parisien, en Ile-de-France et à proximité. Un groupe de départements en sous-réussite un peu moins marquée se situe sur la côte méditerranéenne, des Bouches du Rhône aux Alpes maritimes.

Figure 3

\section{Sur et sous-réussites départementales}

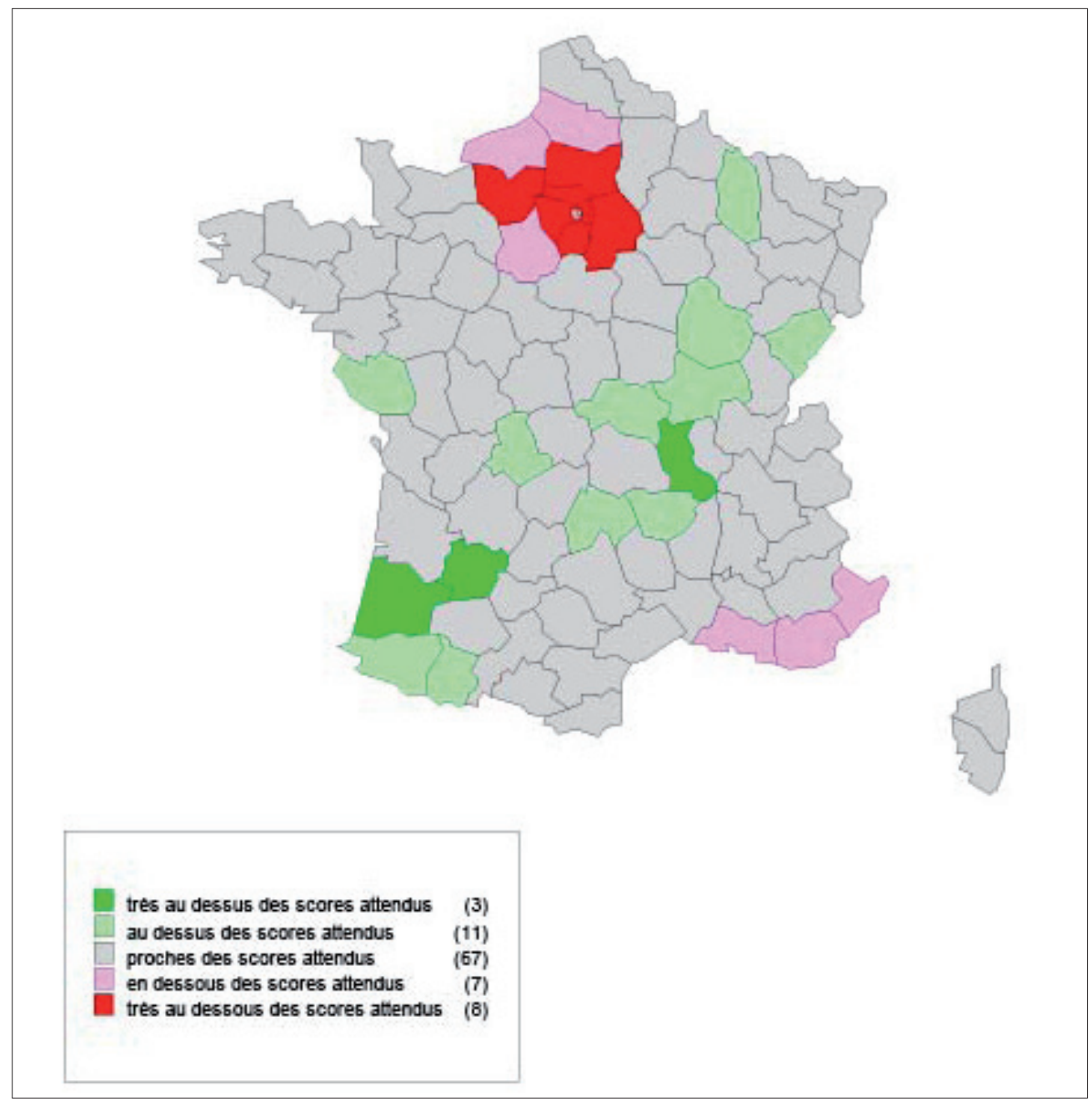

Source et champ : DEPP, ministère de l'Education nationale, Collèges publics de France métropolitaine, évaluation de $6^{\text {ème }}$ (moyenne maths et français, années 2001-2002 et 2003-2004), calculs de l'auteure.

Note de lecture : Les sur ou sous-réussites départementales sont calculées à partir d'un modèle de régression linéaire simple. La variable à expliquer est le score à l'évaluation en $6^{\text {ème }}$ et la variable explicative est l'indice global de précarité qui ne tient compte que des caractéristiques sociales de l'élève. Les résultats observés sont très en-dessous du score prévu ou attendu (sous réussite maximale) en lle-de-France et à proximité. À l'opposé, la sur-réussite est maximale dans les départements de la Loire, des Landes et du Lot-et-Garonne 
Les sur-réussites sont maximales dans le Massif Central, le Sud-ouest, la Loire avec l'agglomération de Saint-Etienne. À l'exception de cette dernière, les départements en sur-réussite ne comportent pas d'agglomération importante alors que les départements où les résultats sont nettement inférieurs à l'attendu sont beaucoup plus urbanisés, ségrégués et se situent, pour la plupart, en Ile-de-France et dans l'extrême Sud-Est méditerranéen. Les disparités et ségrégations entre collèges et les inégalités de réussite selon l'origine sociale y sont exacerbées, tandis qu'elles sont considérablement réduites dans les territoires en sur-réussite, peu urbanisés. Ainsi, ces réussites meilleures ou moins bonnes que prévues sont liées à la tonalité sociale dominante du département et du collège fréquenté, au degré d'urbanisation ou au degré de ségrégation entre collèges dans le département (Broccolichi, Ben Ayed, Trancart, 2005 ; 2010).

En identifiant les caractéristiques des enseignants, on observe qu'ils sont parmi les plus jeunes dans les départements les plus en sous-réussite. Le lien entre jeunesse des enseignants et sous-réussite peut d'ailleurs donner lieu à deux sortes d'interprétation, probablement complémentaires. Une première interprétation consiste à voir le manque d'expérience des très jeunes enseignants comme une cause de sous-réussite. Cependant, la jeunesse des enseignants peut aussi être comprise comme une conséquence de difficultés qui entraînent un départ plus fréquent des plus anciens. Car on sait que l'ancienneté des enseignants affecte lourdement leur possibilité de choisir la région et l'établissement où ils enseignent.

Le cas extrême est celui de la Seine-Saint-Denis, avec $40 \%$ de moins de 30 ans parmi les enseignants des collèges, contre $15 \%$ en moyenne dans les collèges français ces dernières années. Mais on retrouve aussi des pourcentages supérieurs à $35 \%$ dans les zones d'éducation prioritaire (ZEP) de plusieurs autres départements en sous-réussite maximale (Oise, Yvelines, Seine et Marne...) alors qu'ils restent proches de la moyenne nationale dans les ZEP des départements les plus en sur-réussite : la proportion de moins de 30 ans varie ainsi du simple au double entre les départements en sous-réussite maximale et les départements en sur-réussite maximale. On trouve le même lien entre jeunesse des enseignants et sous-réussite quand on considère les résultats des élèves aux épreuves terminales de mathématiques et de français au diplôme national du brevet (DNB), en fin de $3^{\text {ème }}$ et non plus l'évaluation nationale à l'entrée en $6^{\text {ème }}$ : les départements en sous-réussite maximale sont quasiment les mêmes (Broccolichi, Ben Ayed, Trancart, 2005 ; 2010).

En outre, lorsque l'on compare soigneusement leurs performances au DNB, entre 2006 et 2009, selon les départements, les établissements et les groupes sociaux, on constate que ce ne sont pas seulement celles des enfants d'ouvriers et d'inactifs qui sont inférieures aux normes nationales dans les départements urbains et ségrégués. Les élèves des groupes sociaux intermédiaires (employés, professions intermédiaires, artisans et commerçants) y réussissent eux aussi nettement moins bien que leurs homologues des autres départements, et c'est encore le cas (à un degré moindre) des enfants de cadres et d'enseignants (Broccolichi, Sinthon, 2010). 


\subsection{Le tableau noir de l'île-de-France}

Le cas de l'Ile-de-France est particulièrement intéressant. C'est la région où sont concentrés la plupart des départements en sous-réussite maximale à l'entrée et à la sortie du collège, alors qu'elle reste la plus favorisée socialement : le revenu par habitant y est le plus élevé en moyenne ; on y trouve les plus fortes proportions de cadres et de chefs d'entreprise parmi les familles d'élèves, et moins de chômage que dans la plupart des autres régions françaises. Les résultats au $\mathrm{DNB}^{11}$ y sont proches de ceux constatés dans les départements en sous-réussite maximale, mais les inégalités entre les élèves des différents groupes sociaux (et entre établissements) y sont encore plus marquées. Les surcroîts d'échecs et d'inégalités propres à l'Ile-de-France ont retenu l'attention de divers observateurs, au cours des années 90 et du début des années $2000^{12}$. D'autant qu'ils tranchent avec les constats des décennies antérieures. Certes, les évaluations nationales n’existaient pas avant 1989, et les résultats aux épreuves du brevet des collèges n'étaient pas encore centralisés. Néanmoins, toutes les informations disponibles sur les taux d'accès à l'enseignement secondaire puis sur les proportions de diplômés concouraient à faire ressortir l'avance de l'Ile-de-France (et à un degré moindre celle des académies méridionales) sur le reste de la France, avant son déclin relatif par rapport à bon nombre de régions beaucoup moins urbanisées et ségréguées, à partir du milieu des années 80. L'étude de l'évolution sociale des départements d'Ile-deFrance entre les recensements de 1982, 1990 et 1999 (Broccolichi, Ben Ayed, Trancart, 2005) montre clairement une polarisation sociale qui se traduit par une augmentation d'un profil de communes " défavorisées » et une quasi-disparition des communes de profil social « moyen ». Cette polarisation sociale de l'espace résidentiel contribue à accentuer les disparités entre divers établissements.

Globalement, les enquêtes de terrain ${ }^{13}$ montrent que les sous-réussites maximales traduisent l'existence de perturbations des conditions de scolarisation dans de très nombreux établissements. Les cas extrêmes de perturbations associées à des acquisitions scolaires très inférieures à l'attendu résultent souvent de processus cumulatifs : concentrations d'élèves en difficulté, raréfaction des professionnels expérimentés, perte de confiance en l'institution scolaire et évitement croissant des établissements "à risques " par les familles et les personnels de l'éducation. Quant aux sur-réussites, elles sont corrélées à la présence de fortes proportions de professionnels expérimentés, même là où sont scolarisés des publics

11. Les évaluations nationales de Gème et les épreuves du DNB sont construites différemment; on peut cependant noter que les déficits constatés en Ile-de-France à l'entrée en Gème sont proches de $5 \%$ de la moyenne nationale, alors qu'ils sont supérieurs à $10 \%$ de la moyenne nationale pour les épreuves de mathématiques et de français du DNB.

12. Par exemple, l'IAURIF (Institut d'aménagement et d'urbanisme de la Région Ile-de-France) a rédigé, en 2007, un premier rapport de synthèse des travaux sur ces questions, intitulé : Les aspects sociaux de la scolarité en Ile-de-France, puis lui a consacré la "note rapide sur l'éducation et la formation n ${ }^{\circ} 144$ " (6 pages) en mars 2008. Ces deux textes sont consultables sur internet, tout comme les rapports de l'Inspection générale de l'Éducation nationale sur les académies de Créteil, Versailles et Paris qui abordent aussi ces questions.

13. Voir article, rapport et livre cités précédemment. 
d'élèves socialement défavorisés. Elles sont associées également à des continuités, des liaisons et des coopérations qui favorisent la cohérence et la pertinence de pratiques mieux ajustées aux besoins des élèves. En particulier, la stabilité des équipes enseignantes assure une certaine continuité éducative qui permet d'enrayer la spirale de l'échec scolaire.

\section{Conclusion}

Mesurer l'importance relative des fractions de populations scolaires socialement défavorisées selon les territoires à des échelles différentes et repérer dans quelle mesure ces populations se concentrent dans certains espaces urbains ségrégués sont un préalable à l'analyse comparée des performances scolaires. Les départements dont la tonalité sociale est la plus défavorisée sont essentiellement situés dans le nord de la France et en Seine-Saint-Denis. En outre, les ségrégations sociales les plus marquées, avec à la fois des établissements socialement " très favorisés " et "très défavorisés ", sont situés en Ile-de-France mais aussi dans le Sud-est méditerranéen.

Les deux autres principaux résultats qui se dégagent de notre recherche et qui convergent avec ceux de nombreux travaux à différentes échelles (classes, établissements, territoires, pays...) sont, d'une part, que les moins bons résultats scolaires globaux correspondent aux cas où existent les plus fortes disparités ou ségrégations scolaires et sociales (aussi bien entre élèves, qu'entre classes ou établissements) et, d'autre part, que le déficit le plus fort s'observe là où sont concentrés les élèves les plus en difficulté. Ajoutons que ces résultats de type quantitatif prennent davantage sens grâce à une série de travaux qualitatifs sur les liens entre ségrégation, mobilité/captivité et stigmatisation.

Ces conclusions invalident une conception trop mécaniste des relations entre la composition sociale ou même scolaire des publics d'élèves (considérés isolément) et leur réussite scolaire. En particulier, on observe des réussites associées à la présence de personnels expérimentés, même là où sont scolarisés des publics d'élèves défavorisés. Comme le montrent les enquêtes de terrain, les compositions sociale et scolaire des publics d'élèves sont à la fois à distinguer, à replacer dans un espace de comparaisons et de migrations possibles tout en étant aussi à relier à d'autres composantes des conditions de scolarisation avec lesquelles elles peuvent interagir selon des configurations très variables.

\section{Bibliographie}

Barthon C. (1997), «Enfants d'immigrés dans la division sociale et scolaire », Les annales de la recherche urbaine, $\mathrm{n}^{\circ} 75$, pp. 70-78.

Broccolichi S. (2011), "L'espace des inégalités scolaires ", Actes de la recherche en sciences sociales, $\mathrm{n}^{\circ} 179$, pp. 4-13. 
Broccolichi S. et van ZANTEN A. (1997), « Espaces de concurrence et circuits de scolarisation. L'évitement des collèges publics d'un district de la région parisienne ", Les annales de la recherche urbaine, $\mathrm{n}^{\circ}$ 75, pp. 5-17.

Broccolichi S., Ben Ayed C., Trancart D. (coord) (2005), Les inégalités socio-spatiales d'éducation, Rapport réalisé pour la DEPP.

Broccolichi S., Ben Ayed C., Trancart D. (coord) (2010), Ecole : les pièges de la concurrence. Comprendre le déclin de l'école française, La Découverte.

Broccolichi S., Sinthon R. (2010), « Libre choix, hiérarchisation des espaces scolaires et surcroît d'échecs ", in Ben Ayed C. (Dir.), L'Ecole démocratique, Armand Colin, pp. 160-173.

Duncan 0. et Duncan B. (1955), "Methodological analysis of segregation indexes", American Sociological Review, $\mathrm{n}^{\circ} 20$.

Duru-Bellat M. (2004), Les effets de la ségrégation sociale de l'environnement scolaire : l'éclairage de la recherche, étude réalisée à la demande de la Commission du débat national sur l'avenir de l'École.

Duru-Bellat M., Landrier-Le Bastard S., Piquée C. (2004), "Tonalité sociale du contexte et expérience scolaire des élèves au lycée et à l'école primaire ", Revue française de sociologie, $\mathrm{n}^{\circ} 3$, pp. 441-468.

Felouzis G. (2005), L'apartheid scolaire : Enquête sur la ségrégation ethnique dans les collèges, Paris, Édition du Seuil (avec F. Liot et J. Perroton).

Meuret D., Broccolichi S., Duru-Bellat M. (2001), "Autonomie et choix des établissements scolaires : finalités, modalités, effets ", cahiers de l'Iredu, n 62.

Maurin E. (2004), Le ghetto français. Enquête sur le séparatisme social, Paris, Éditions du Seuil.

Poupeau F., François J.-C. (2008), Le Sens du placement. Ségrégation résidentielle et ségrégation scolaire, Paris, Raisons d'agir.

PréiteCeIlle E. (2003), La division sociale de l'espace francilien. Typologie socioprofessionnelle 1999 et transformation de l'espace résidentiel 1990,1999, Paris, OSC, FNSP, CNRS.

Préteceille E. (2006), « La ségrégation sociale a-t-elle augmenté ? La métropole parisienne entre polarisation et mixité ", Sociétés contemporaines, n 62, pp. 69-93.

Préteceille E. (2009), " La ségrégation éthno-raciale a-t-elle augmenté dans la métropole parisienne ", Revue française de sociologie, vol. 50, n³ 3, pp. 489-519.

Rhein C., (1997), “ De l'anamorphose en démographie. Polarisation sociale et flux scolaire dans la métropole parisienne ", Les annales de la recherche urbaine $\mathrm{n}^{\circ}$ 75, pp. 59-69. 
TABARD N. (1993), « Représentation socio-économique du territoire, Typologie des quartiers et communes selon la profession et l'activité économique de leurs habitants ", France métropolitaine, recensement de 1990, INSEE.

TABARD N. (2002), « Représentation socio-économique du territoire, Typologie des quartiers et communes selon la profession et l'activité économique de leurs habitants ", France métropolitaine, recensement de 1999, INSEE.

Trancart D. (1998), "L'évolution des disparités entre collèges publics ", Revue française de pédagogie, $\mathrm{n}^{\circ} 124$, pp. 43-53.

VAn Zanten A. (2000), "De la diversité à la ségrégation scolaire » in Dupuis P.-A, L'école en devenir, l'école en débat, l'Harmattan, pp. 15-27. 